\title{
Acercamiento al uso de Bots como interfaz para Internet de las Cosas
}

DOI: $10.46932 / \operatorname{sfjdv2n1-083~}$

Received in: November 1st, 2020

Accepted in: December 30th, 2020

\section{José Manuel Inestroza Murillo}

MSc. Gestión de Tecnologías de Información, Ing. en Sistemas

Departamento de Ingeniería en Sistemas, Facultad de Ingeniería, UNAH

Ciudad Universitaria, Blv. Suyapa, Tegucigalpa, Honduras , C.A.

E-mail: jose.inestroza@unah.edu.hn

\section{RESUMEN}

Los sistemas embebidos y computadores de una sola placa nos ofrecen conectividad de aparatos domésticos para utilizarlos de forma conveniente pero que dependen de múltiples interfaces de comunicación independientes, cada una de acuerdo con las necesidades de cada fabricante. Aprovechando el modelo de envío de mensaje de textos para establecer comunicación entre dos personas, podemos hacer uso de los ChatBots (Bots o programas informáticos que se comunican mediante texto) para intercambiar información entre dos actores, siendo el primero un humano que genera instrucciones y el segundo un programa máquina que recibe, interpreta y ejecuta dichas instrucciones. De esta forma es posible reemplazar cada interfaz independiente por una única interfaz de texto a través de un Bot para enviar y recibir instrucciones para el control de múltiples periféricos de Internet de las Cosas.

Palavras chaves: Bots, ChatBots, Lenguaje Natural, Reconocimiento de Patrones,Internet de las Cosas,Telegram,IFTTT.

\section{INTRODUCCIÓN}

El modelo de comunicación de instrucciones a través del lenguaje natural sobre las computadoras es un concepto asociado comúnmente con la idea de que una máquina deba ser capaz de hablar por si misma, usando "inteligencia" para la comprensión del habla y/o la escritura, y al mismo tiempo conocimiento del lenguaje y su significado para poder realizar una respuesta como contraparte a la comunicación o petición original. Algunas de estas nociones son tan antiguas como las definidas por Allan Turing en su artículo original de “El juego de imitar” en la década de los 50’s [1].

No obstante, la complejidad que yace detrás de un sistema capaz de comprender la semántica de nuestras oraciones, de identificar el contexto, asociar o investigar los lexemas de manera inteligente y sobrepasar las deficiencias gramaticales sin perder el significado general del texto percibido (así como lo hacen los humanos de forma natural) es un tema que aún necesita desarrollo bajo ramas de estudio como redes neuronales, aprendizaje máquina, comprensión de lenguaje, inteligencia artificial en general y ramas afines. No debemos descartar que el uso de lenguaje natural para entregar instrucciones escritas o habladas a las máquinas es parte de tecnologías comerciales de dispositivos o servicios que pueden ser utilizados 
para hacer búsquedas en internet, para el manejo de agenda, recordatorios o calendarios de actividades [2]. Entre estos nos encontramos con servicios como: Google Assistant, Amazon Alexa, Microsoft Cortana y Apple Siri, por mencionar algunos, son asistentes digitales que se encuentran en aplicaciones de escritorio, dispositivos inteligentes y en dispositivos móviles.

La idea de generar una interface unificada que pueda centralizar instrucciones es por tanto algo que ya es parte de sistemas informáticos públicos o personales, que utilizan servicios privados en la nube y que inclusive ofrecen a usuarios aprovechar dichas plataformas para intereses domésticos y comerciales. Algunos de estos productos "modernos" pueden destacarse bajo la categoría de "parlantes inteligentes" (smart speakers) pero también aplican los buscadores en línea (web search engines), entre otros. Muchos de estos dispositivos pueden accederse por tanto únicamente por voz o a través del App propia del fabricante para configurar dicho dispositivo.

Además, existe la posibilidad de que los usuarios deseen mantener en privacidad la generación de instrucciones en lugar de comunicar dichos mensajes en "voz alta" en espacios abiertos donde otros usuarios puedan escuchar lo que dictamos, o inclusive necesitar un agente que simplemente pueda "ver" y comunicarse con múltiples dispositivos en lugar de mantener una comunicación con un App separada por cada dispositivo final. Es de esta forma que el texto puede convertirse en una alternativa a la voz para establecer comunicación con dispositivos IoT (Internet of Things, por sus siglas en inglés), y hacer uso de los Bots o Chatbots como una plataforma intermedia para comunicar nuestras instrucciones a dispositivos propios previamente configurados.

\section{SOBRE LA PROBLEMÁTICA}

Existe un patrón para el uso de dispositivos o servicios donde cada fabricante se encarga de generar una interfaz que puede ser física o virtual que lo destaca sobre el resto de la competencia y que representa el panel de control entre el usuario y la funcionalidad deseada, sea esta electrónica, mecánica o eléctrica. Debido al creciente número de dispositivos que hoy en día pueden conectarse a Internet (focos, parlantes, relojes, teléfonos, televisores, refrigeradores, entre otros), los usuarios pueden enfrentarse a un abrumador escenario donde deben controlar numerosas y diferentes aplicaciones de forma independiente y aislada para manipular o mantener sus dispositivos de internet de las cosas.

Es por ello que se pretende en este informe hacer uso del concepto de las plataformas de Bot para implementar una unificación de múltiples dispositivos para que puedan comunicarse desde una única interfaz de texto mediante un agente programable, controlado desde un servicio intermedio en Internet para enviar instrucciones remotas a nuestros propios dispositivos en casa, sean estos últimos fabricados por nosotros mismos o por fabricantes terceros. 
Debido a las similitudes y características compartidas entre distintas plataformas Bot en línea que comparten el propósito conceptual del manejo de conversaciones, este documento se aprovecha de las cualidades del uso de una plataforma Bot para demostrar el funcionamiento del acercamiento propuesto de "...Bots como interfaz para el internet de las cosas".

El objetivo principal por tanto será explorar el uso de un Bot como principal medio de interacción entre periféricos, aplicando investigación cualitativa para entregar una visión subjetiva de la exploración del fenómeno planteado, manteniendo como alcance primario la identificación de los componentes de hardware y software requeridos para comprobar la viabilidad del acercamiento.

\section{COMPONENTES DE HARDWARE PARA COMUNICACIÓN DE PERIFERICOS}

Para poder implementar un modelo de unificación de dispositivos propios que conversan a través de Internet, debemos considerar los distintos componentes eléctricos y electrónicos que nos permitirán conectarnos con los periféricos deseados antes de construir nuestra propia red de comunicaciones y procesamiento de instrucciones.

Se espera que las secciones siguientes permitan al lector formarse una secuencia de los componentes, partes y actores que se requieren para poder comunicar los componentes físicos y hacer uso de ellos mediante servicios digitales.

\section{A. Sistemas Embebidos}

IoT es un concepto relacionado con la conectividad de todos los objetos o dispositivos que de manera cotidiana están involucrados en las actividades de las personas para que, a través de la recopilación masiva de datos y su conectividad a internet de manera autónoma, podamos conocer información de consumo y hábitos de los usuarios [11]. Por otra parte, podemos hacer disposición de sistemas embebidos que son componentes computarizados construidos con un propósito específico donde sus partes se manufacturan usualmente para ser más pequeños enfocándose en el ahorro de energía y fabricados para integrarse con otros componentes [5] [6].

Uno de los usos de los sistemas embebidos asociado a IoT es comunicar dispositivos de la vida cotidiana que no están interconectados en la nube para que puedan activarse o desactivarse a través de otros aparatos que sí lo están, indistintamente si los primeros son eléctricos, electrónicos o mecánicos. Llamaremos a estos dispositivos finales como periféricos y consideraremos como ejemplo de acciones sobre dichos periféricos actividades como: activar las conexiones eléctricas de iluminación de una casa, provocar el encendido de un vehículo, habilitar un ventilador de sala, iniciar la grabación de un audio o video, entre otros. Mediante componentes eléctricos y electrónicos, por tanto, debemos re-plantear la 
posibilidad de interactuar de forma remota y digital sobre estos periféricos cotidianos.

\section{B. Computadoras de una sola Placa}

Existen componentes electrónicos más complejos que representan computadores de tamaño reducido conocidos como Computadores de una sola Placa o SMC (Single Board Computer, por sus siglas en inglés) [7], los cuales se compone en su mayoría por un SoC (System on a Chip), capaces de contener múltiples componentes electrónicos en un sólo circuito integrado y que pueden realizar acciones más complejas debido a una mayor compatibilidad con puertos, protocolos y estándares de comunicación. Algunos ejemplares SMC conocidos por sus marcas comerciales son: Raspberry Pi, Arduino y Beaglebone. Los SMC usualmente poseen una interfaz genérica de interconexión GPIO para generar alimentación a otros componentes electrónicos externos (General Purpose Input Ouput, por sus siglas en inglés) que extienden la funcionalidad del computador sobre escenarios más especializados o específicos no contemplados inicialmente por el fabricante de la placa.

También, de manera esencial, debemos considerar el componente interruptor o relevador relay para permitir el paso o interrupción de corriente, el cual controla la activación (permisividad) o desactivación (anulación) de energía desde una fuente eléctrica hacia otro dispositivo externo, de tal forma que haciendo uso de diseño de circuitos electrónicos sea posible generar una placa adicional de componentes que pueda recibir una instrucción digital remota y traducir esta instrucción hacia el mundo físico provocando el encendido o apagado de un periférico.

Observe la Fig. 1 como un ejemplar de interconexión entre un SBC y un módulo de 8 relay’s interconectados por jumpers simbólicos a través del puerto GPIO de una Raspberry Pi con configuración $\mathrm{A}+, \mathrm{B}+\mathrm{o}$ Pi 2 .

Figura 1. SBC Raspberry Pi conectado con un módulo de 5 Voltios 8 Relay’s

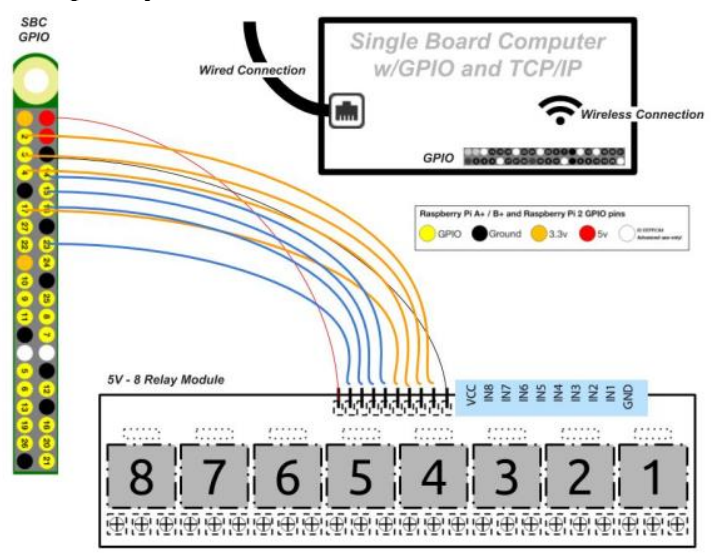




\section{COMPONENTES DE SOFTWARE PARA IMPLEMENTACIÓN DE LA COMUNICACIÓN}

\section{A. $\quad$ Modelo de Comunicación entre Plataformas}

Considere que la comunicación entre los componentes se reduce a una lógica de 3 pasos cíclicos que nacen y finalizan en el dispositivo del paso 1 pasando por los intermediarios paso 2 y paso 3 , y que cada paso corresponde con un actor o agente siguiente: 1) Componente dispositivo móvil con cliente Bot, que refiere a la interfaz que interactúa con el usuario, es decir “el Bot”, la cual puede ser un app móvil, un sitio web, un programa de computadora de escritorio, entre otros, que incluye un chat como un medio para enviar y recibir mensajes de texto; 2) Componente servidor API Bot, el cual es la infraestructura que está detrás del Bot como un servicio en internet que posee una Interfaz de Programación de Aplicaciones (Application Programming Interface o API por sus siglas en inglés) y es quien recibe la consulta de texto desde el agente 1 y que re-direcciona la petición hacia un servicio web externo a la plataforma del Bot que conoceremos como agente 3 utilizando la conceptualización del webhook (anclaje web) o forwarding (re-direccionamiento), que hace el push hacia un tercero re-enviando la petición original (request); 3) Componente servicio BackEnd para el procesamiento de instrucciones o comandos, disponible como un servicio privado en internet dentro de un host de acceso público, quien recibe el comando re-direccionado por el agente 2 y que al inicio, durante o finalizado su proceso interno comunica una o múltiples respuestas o acciones hacia el agente 1 o hacia los dispositivos periféricos conectados; suele notificar mediante retroalimentación de texto el éxito o fracaso del proceso.

La interacción completa de mensajes entre plataformas se realiza a través de la comunicación HTTP POST y GET usando nomenclatura JSON para el paso de datos (Javascript Object Notation o Notación de Objetos en lenguaje Javascript por su significado en español), mientras que la comunicación con los dispositivos finales se realiza a través de sus propias interfaces o API's.

\section{B. Lógica del Servicio BackEnd}

El servicio BackEnd consiste en una plataforma web capaz de recibir las peticiones HTTP POST request con la consulta original serializada en formato JSON con la parametrización de acuerdo con la configuración del Componente Servidor API Bot. Este servicio toma la instrucción de lenguaje natural y descompone la oración para que esta sea procesada de acuerdo con la secuencia de condiciones que permitirán ejecutar una acción deseada que corresponde con el significado de la instrucción entrante. De esta forma el BackEnd debe contener en su lógica un diccionario de comandos o instrucciones y su correlativo de acción a ejecutar. 
Figura 2. Diagrama de Secuencia Lógica de la Comunicación de Componentes a Alto Nivel.

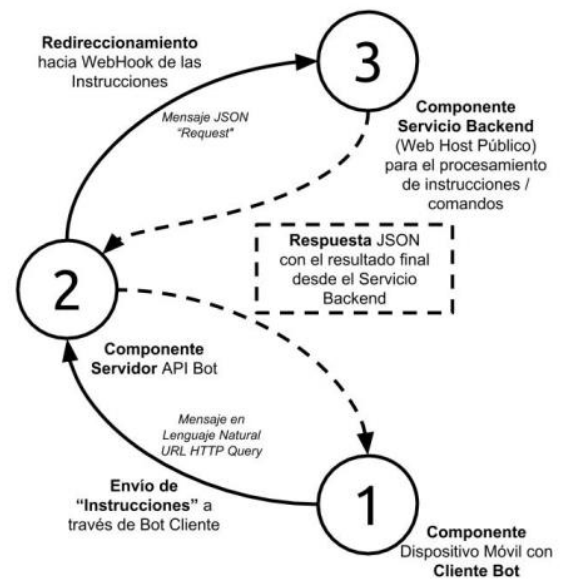

Desde el punto de vista de ingeniería de software el componente de verificación de comandos debe reducir en cascada las posibles alternativas de comandos a reconocer para descartar la evaluación de palabras innecesarias. Analizaremos a continuación dos formas procedurales y una tercera forma mediante inteligencia artificial para implementar el procesamiento de las instrucciones en lenguaje natural (NLP o Natural Language Processing por sus siglas en inglés). De acuerdo con [8] las palabras poseen tres tipos de expresión: ente (entity, individuo), evento (event) y funcionales (functional); de forma general los nombres propios y adjetivos invocan a los "entities", los verbos y adverbios invocan a los "events", los términos generales (common nouns) invocan a las "entities" y "events", y finalmente el resto de palabras invocan las expresiones "functional".

Aunque este estudio no profundiza en la construcción lingüística de la semántica de la instrucción en lenguaje natural, considera que cada texto debe estar compuesto de un orden secuencial de palabras claves que a su vez pueden tener sinónimos y que por tanto corresponden con un árbol o grafo compuesto de nodos, donde cada nodo representa una palabra clave, y donde cada palabra clave se ordena una detrás de otra para que en conjunto conformen una "acción final" a ser interpretada por el servicio detrás. 
Figura 3. Lógica del servicio BackEnd Agente no. 3 para el Procesamiento de Instrucciones en Lenguaje Natural.

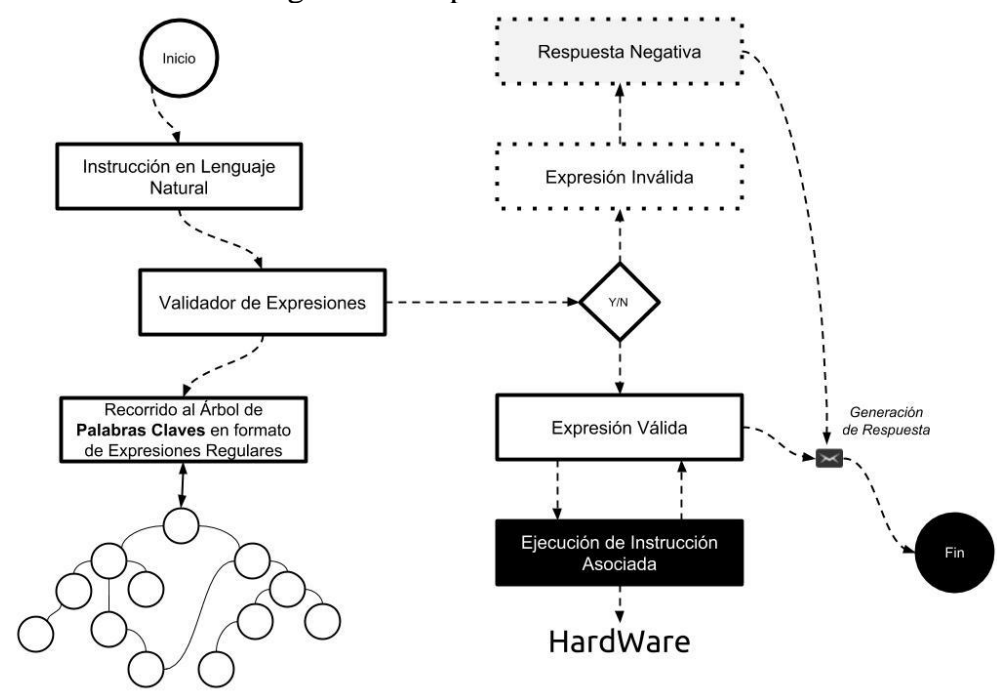

Considere por ejemplo la secuencia de palabras: "toma fotografía sala" y sus variantes similares "envía foto sala", "enviar imagen sala", "enviar fotos sala", entre otras combinaciones en plural o singular que corresponden con la misma semántica. Observe además que una oración cualquiera como es "Enviarme fotografías de la sala", cumple con la secuencia antes mencionada de palabras, resaltando en negrita y subrayado las palabras que corresponden con la secuencia en mención sin considerar el resto de componentes formales de la gramática. Cada una de estas secuencias, por tanto, debe representarse por un grafo compuesto de nodos (un nodo por palabra), usando cada palabra única en una posición específica y una tras de otra para conformar un orden. En total podemos observar que el conjunto de opciones para la palabra de la posición 1 corresponde con la lista "toma, envía" (ignorando posteriormente las tildes para considerar incrustación del texto dentro de otras palabras) y así palabras como "enviar", "enviarme" y "envía” cumplirán con la secuencia de letras de “e,n,v,i,a”, ya sea por una comparación exacta o por una inclusión/incrustación de la palabra base dentro de la palabra a comparar (haciendo referencia a un contains o "contenido en" dentro de programación).

Esta secuencia de patrones también es realizable mediante la implementación de expresiones regulares, las cuales hacen uso de autómatas finitos para identificar una secuencia cerrada de combinaciones de letras, una tras de otra, para finalmente corresponder con un patrón o estructura de palabras en común. Por ejemplo, las secuencias numéricas “123”, “2”, “4.0”, “3.14” corresponden en su totalidad con el patrón de un número (real o entero) que en expresiones regulares podrán representarse con la simbología “ld+( (\.|d+)?” que coincide con el orden: “un dígito numérico repetido una o más veces, seguido opcionalmente de un punto decimal que cuando existe obligatoriamente es seguido de uno o más dígitos numéricos". Esta nomenclatura de expresiones regulares se compone de cuantificadores, reglas y definiciones que permiten en combinación identificar patrones complejos de cadenas de texto. 
Haciendo uso de los patrones en mención se ha desarrollado una expresión que es compatible con todas las alternativas definidas para la palabra de la posición 1 (asumiendo una comparación exacta usando únicamente letras minúsculas) que se lee como: “(toma(r)?)|(env[ií]a(r)?)". La expresión anterior corresponde con un match para las palabras "tomar", "toma", “envía”, "enviar", sin embargo, esta expresión no corresponde en comparación con otras variantes de estas palabras como ser "enviarme" o “tomad". Para ello, la comparación de la oración final deberá contener un patrón más complejo que considere escenarios múltiples.

Vea el siguiente patrón: “.*((toma)|(env[ií]a)).*((foto)|(im[aá]gen)).*(sala).*”. Este patrón permite contemplar cualquier otra palabra o símbolo al inicio, final o a mitad de las palabras claves definidas (incluyendo derivaciones como "tomar" - "fotografía” - "imágenes” que no están explícitamente escritas en la expresión regular). Finalmente, una vez identificado el patrón genérico, debemos indicar al computador la acción a asociar para con esta secuencia de palabras. Para el ejemplo en curso el texto simplemente corresponde con "hacer uso de la cámara de seguridad de la sala de su hogar, tomar una fotografía y enviarla de regreso al chat del usuario original”. Para ejecutar esta acción el implementador deberá hacer uso del API del fabricante de la cámara para tomar la fotografía, y luego hacer uso del API del Bot para enviar la imagen al usuario.

También podemos considerar machine learning (ML) o aprendizaje máquina para el NLP de las instrucciones al alimentar una red neuronal artificial (ARN o Artificial Neural Network por sus siglas en inglés) con frases u oraciones que contengan significados comprobados en la vida real y que correspondan con la semántica real de la acción que se quiere realizar. Esto significa que, por ejemplo, se debe entrenar una red para que comprenda como 1000 frases (en el mismo idioma), significan la misma acción de tal forma que cuando la red reciba una oración que nunca había visto antes pero que es congruente con el mismo significado (porque hace uso de palabras similares en posiciones usuales o actores o verbos en común con el resto de oraciones). Entonces, si la red es entrenada correctamente usando suficientes escenarios de éxito o fracaso, la misma podrá encontrar la similitud dentro de la nueva frase y por tanto ejecutará la acción deseada en conformidad [13].

El lector debe estar al tanto que los mecanismos expuestos de árboles de decisión o el uso de expresiones regulares no son infalibles, ya que semánticamente hablando, para el programa de computadora significa exactamente lo mismo la secuencia de letras "tomafotosala" (la cual es una oración gramaticalmente incompleta) como también "computadora, tomar una fotografía de la sala y enviarla al correo electrónico", tal y como se lo podríamos dictar a un asistente digital como por ejemplo Cortana, Google Assitant o Amazon Alexa, entre otros. Una vez recibida y analizada la expresión, si ésta es aceptada o rechazada, la plataforma BackEnd podría generar una retroalimentación de éxito o fracaso al 
usuario final.

\section{Ejección de Instrucciones en el Hardware}

Una vez identificada la acción correcta, el servicio BackEnd debe comunicarse con el dispositivo o conector inteligente específico entre toda la red de periféricos conectados o autorizados a ser "visibles" a través del BackEnd.

Al conectar los dispositivos a través de internet, los servicios serán capaces de realizar envío de mensajes in situ o de forma remota siempre que la conexión esté disponible.

En la dirección de instrucciones, el mecanismo de Pull de Mensajes corresponde con servicios de escucha que deben consultar con frecuencia al Servidor para saber si existen nuevas instrucciones. Esto consume recursos y requiere de trabajos que se deban ejecutar cada cierto tiempo en búsqueda de nuevas peticiones. Caso contrario, en el mecanismo Push de Mensajes desde el BackEnd Service en la nube promueve la llamada del servicio Cliente generando la solicitud original desde el Servidor y propagando el mensaje hacia los clientes en escucha.

Figura 4. Lógica de detección y recepción de instrucciones desde SBC.
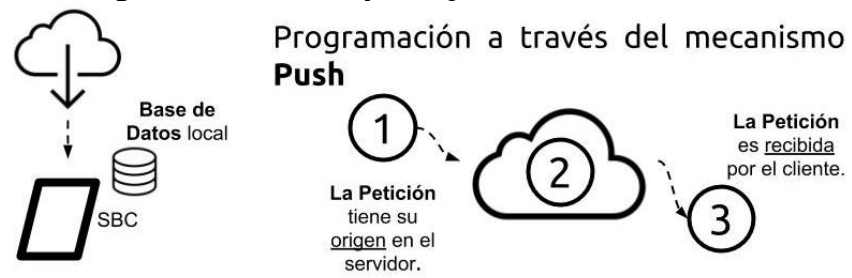

Programación a través del mecanismo Pull

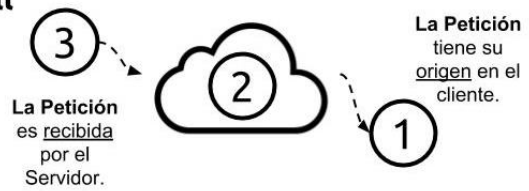

En la Fig. 5 veremos un ejemplar de integración entre el servicio BackEnd en línea para comunicarse a través de un motor de base de datos u otra plataforma de intermediación para con los dispositivos personales wearables (usables por las personas), home appliances (aparatos domésticos en casa) u otros. 
Figura 5. Lógica de interconexión entre Hardware.

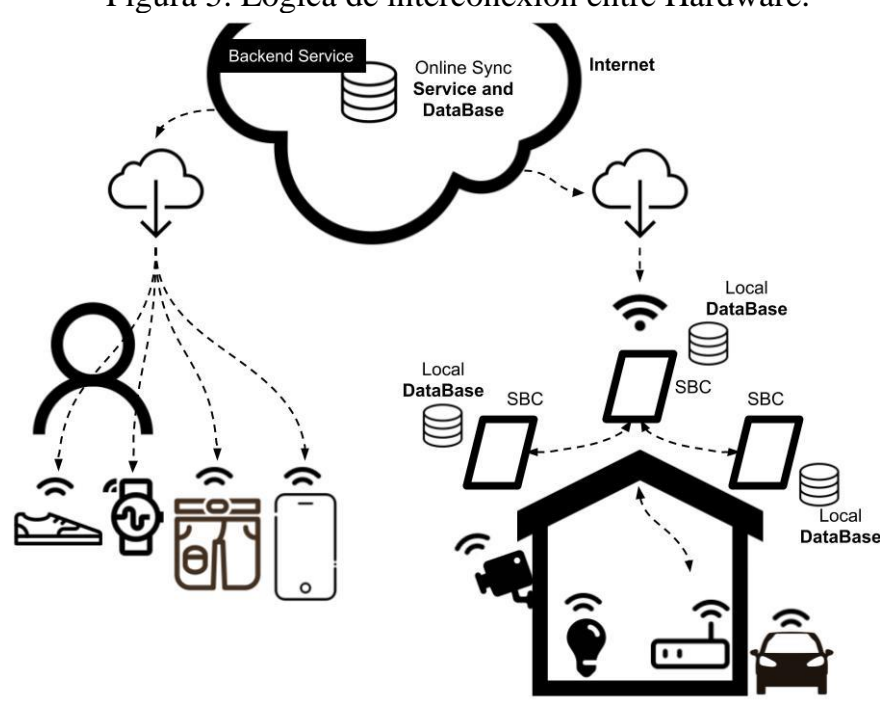

De esta forma todos los dispositivos o servicios que deban recibir instrucciones desde el BackEnd deben contener un componente intermediario con un mecanismo de consulta y/o un mecanismo de escucha que les permita buscar o recibir las tareas a ejecutar.

\section{Variante de dispositivos interconectados}

Existen diferencias en los tipos actuales y rubros de los objetos que pueden ser parte de IoT [11]. Observe la categorización de la Fig. 6. Este estudio se ha considerado tres alcances generales de esta visión universal de conectividad hacia Internet.

En la ejecución de comandos para conocer el estado de dispositivos en casa (home status and commands execution) es posible incluir (sin limitarse a) la siguiente lista de posibles periféricos: sistemas de iluminación, equipos de monitoreo de temperatura, cámaras de seguridad y vigilancia, aparatos inteligentes domésticos y cualquier dispositivo que sea controlado a través de conectores inteligentes con relay's integrados para el paso de corriente. Entre los escenarios de uso podemos identificar casos como: "Activar el ventilador o el aire acondicionado del dormitorio o cambiar la temperatura según se desee", "Encender el horno como preparación a una comida", “Apagar las luces de toda la casa desde el dormitorio cuando se está a punto de ir a dormir" o "Tomar una fotografía del estado actual de todas las cámaras de seguridad de la casa".

También se pueden considerar una categoría de dispositivos inteligentes de uso personal y de vestimenta (smart gadgets and wearables) incluyendo pero no limitándose a: relojes, ropa, cinturones, botellas para agua, calzado y demás posibles accesorios inteligentes (incluyendo smartphones). En general estos dispositivos pueden recolectar información a diario sobre nuestros hábitos, actividad física como peso o ritmo cardiaco, o sobre el estado en general del usuario que los utiliza. 
Por último podemos considerar otros dispositivos conectados a IoT (related to IoT), sobre todo para la ejecución instrucciones remotas, incluyendo pero no limitándose a: vehículos inteligentes, seguridad y protección, seguimiento de mascotas y miembros de la familia.

Aunque en esta investigación no se profundizará sobre temas éticos o de privacidad, los asuntos de seguridad y protección [11] están asociados, por ejemplo, a saber de forma remota si el horno de la estufa en nuestra casa está apagado o accionar el apagado en caso que sea necesario (como prevención de accidentes domésticos), o en el seguimiento de mascotas o miembros de la familia, asociado a escenarios donde se requiere conocer la ubicación de un menor durante un viaje de escuela o sobre la localidad de un adulto mayor que requiere de seguimiento de un familiar que está de viaje.

Figura 6. Unificación de estados y consultas a servicios y dispositivos IoT a través de un Bot usando Lenguaje Natural.

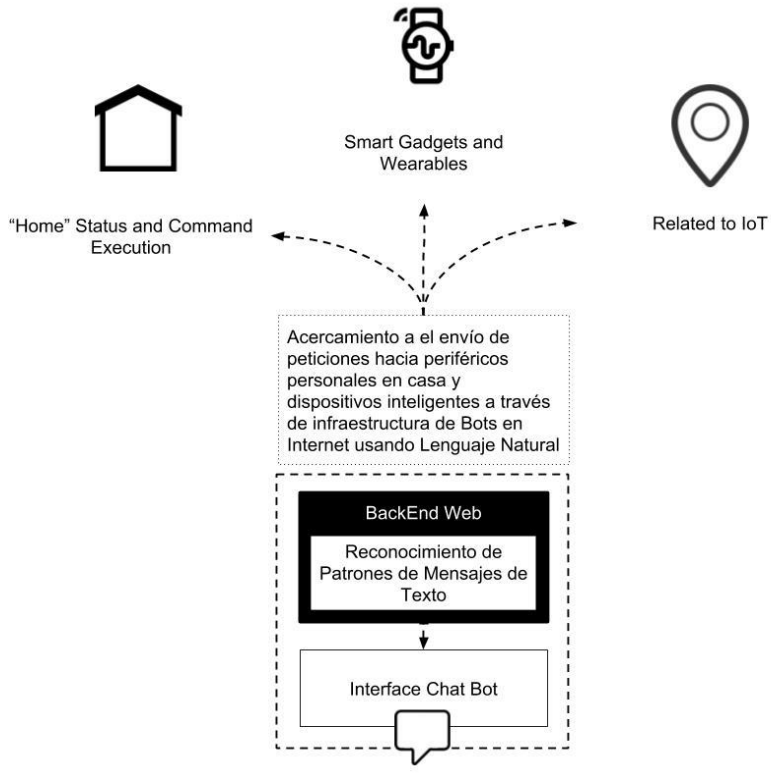

Además, el usuario implementador debe considerar que es posible que algunas de las plataformas deban conectarse utilizando un único mecanismo o protocolo de mensajería privativo, limitándose a la funcionalidad que el fabricante otorga al usuario final. 
Figura 7. Mecanismo integrado de notificaciones dependiendo del dispositivo. Ejemplo a través de Google Cloud Message.

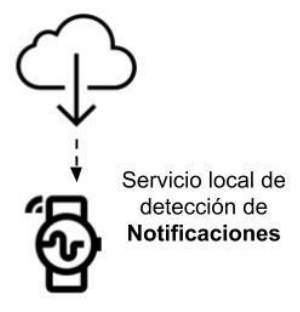

Mecanismo Integrado de Notificaciones

(ej. Google Cloud Message - GCM)

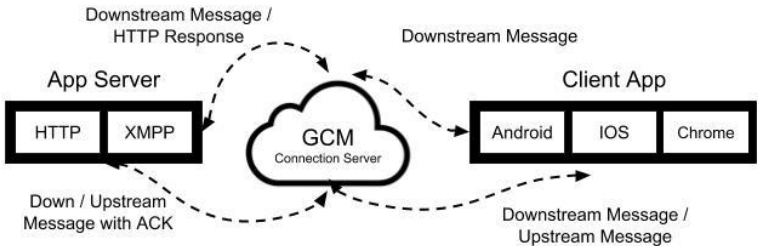

\section{E. Conexión Remota}

Aunque el futuro de IoT apunta a una conectividad enorme de dispositivos, para el momento y lugar de la definición de este documento, aún existen limitaciones en la comunicación alámbrica o inalámbrica entre aparatos, como por ejemplo sobre aquellos relojes inteligentes que solo pueden comunicarse por Bluetooth, o entre Electrodomésticos que no cuentan con una interfaz inteligente y que requieren de una adaptador especial para interactuar físicamente con el dispositivo. Es por tanto que de acuerdo con las limitantes de Hardware (chips de comunicación, puertos físicos) y Software (API's, puertos virtuales, protocolos, sistemas operativos) el usuario tendrá que establecer sus propias interfaces intermedias en el dispositivo aprovechando desde software libre (opensource) y servicios gratuitos en línea hasta soluciones de paga o de software privativo, y deberá conectar esas interfaces mediante el protocolo correcto hacia el BackEnd en internet, y de acuerdo con las ventajas y limitantes encontradas, entonces diseñará una solución óptima y personalizada para el usuario implementador.

Figura 8. Enfoque de dispositivos siempre conectados

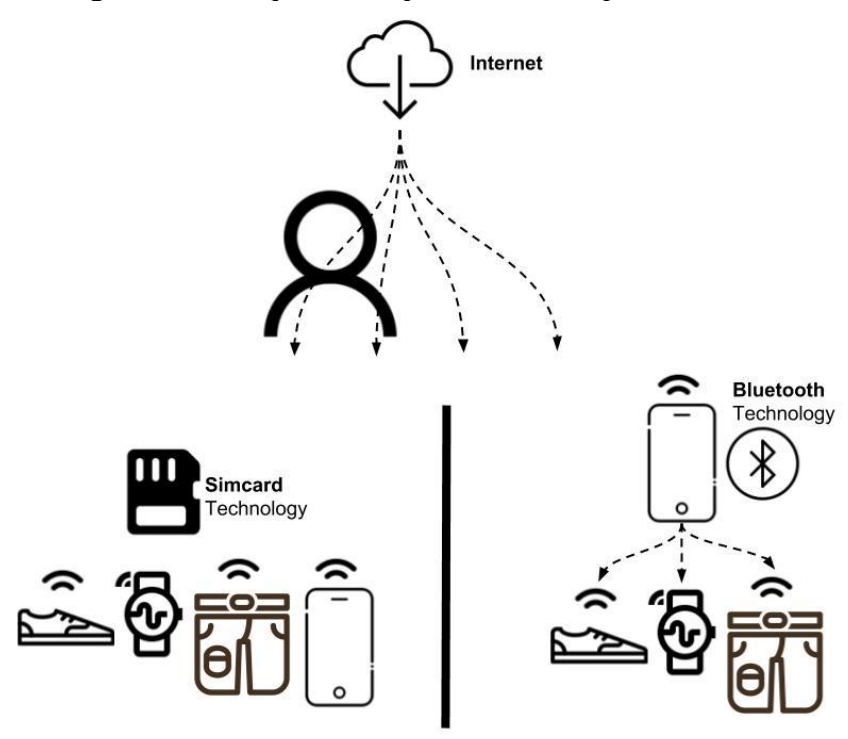




\section{PROPUESTA DE EXPERIMENTO}

Para demostrar los elementos planteados en este estudio se ha realizado un prototipo como experimento incompleto pero funcional que integra Bots, un servidor web público y un computador para simular la lógica de recepción del comando en todos sus flujos e integrándose con otras plataformas en línea para un uso más extenso en la temática de IoT.

\section{F. Telegram como Plataforma Bot}

Como investigadores, implementadores y usuarios finales de servicios en internet podemos hacer uso de cualquier plataforma según conveniencia para la experimentación e implementación de lo descrito en este documento, para comunicar nuestros dispositivos personales, periféricos en casa y otros, y hacer una verdadera interconexión de gadgets a través de una única interfaz de texto.

Así como "Whatsapp”, “Facebook Messenger”, “Google Hangouts”, “Apple Messenger”, entre otros, "Telegram" es un servicio de mensajería en línea que tiene una plataforma de Bots con documentación para el uso de su API [9] que nos permitirán crear un robot de texto con un nombre propio (utilizando el componente conocido como BotFather de Teleram) que puede usarse en chats con comunicación de uno a uno, que puede incluirse en grupos de muchas personas dentro de la misma plataforma de Telegram, que integra un redireccionamiento o anclaje web (WebHook) desde el Bot Server hacia un servidor web tercero cualquiera para el procesamiento del texto, y que permite a través de sus mensajes comunicar texto, audio, imágenes y videos (incluyendo streaming) dentro de las respuestas del bot hacia el o los usuarios a quién sirve.

Es esta plataforma Bot la que recibe los mensajes de texto enviados desde el Chat (desde una aplicación móvil o desde una interfaz web), los cuales redireccionará hacia un WebHost Server público en internet en el cual deberá hospedar la plataforma BackEnd Web. Esta plataforma puede ser un servidor Web cualquiera como ser Ant, IIS, GlassFish, etc, y debe tener una dirección pública con la cual se pueda hacer el llamado del WebHook. La lógica de programación, el lenguaje y el procesamiento en general que haga este servicio dependerá de las propias necesidades del usuario.

Para el experimento base se ha utilizado el lenguaje PHP a través de un servidor Apache2 para recibir las instrucciones del Request POST del WebHook y luego se han escrito las instrucciones ya traducidas o interpretadas como acciones en una tupla de base de datos, usando un motor de base de datos MySQL, y luego utilizando una tarea programada dentro de un computador linux mediante CRONJOB's para provocar un PUSH que identifique si hay instrucciones en la tabla, se ha detectado el comando pendiente de atender. De esta forma si conectamos este programa a una placa Rasberry Pi, podríamos usar un lenguaje de alto nivel como Python para hacer uso de los puertos de propósito múltiple GPIO para enviar la instrucción de paso de energía sobre un LED por ejemplo para activar un pequeño indicador de 
éxito, o sobre un Relay para permitir la activación de una lámpara, etc. La complejidad de la solución dependerá de la magnitud del problema.

Observe la Fig. 9 para visualizar el flujo completo final.

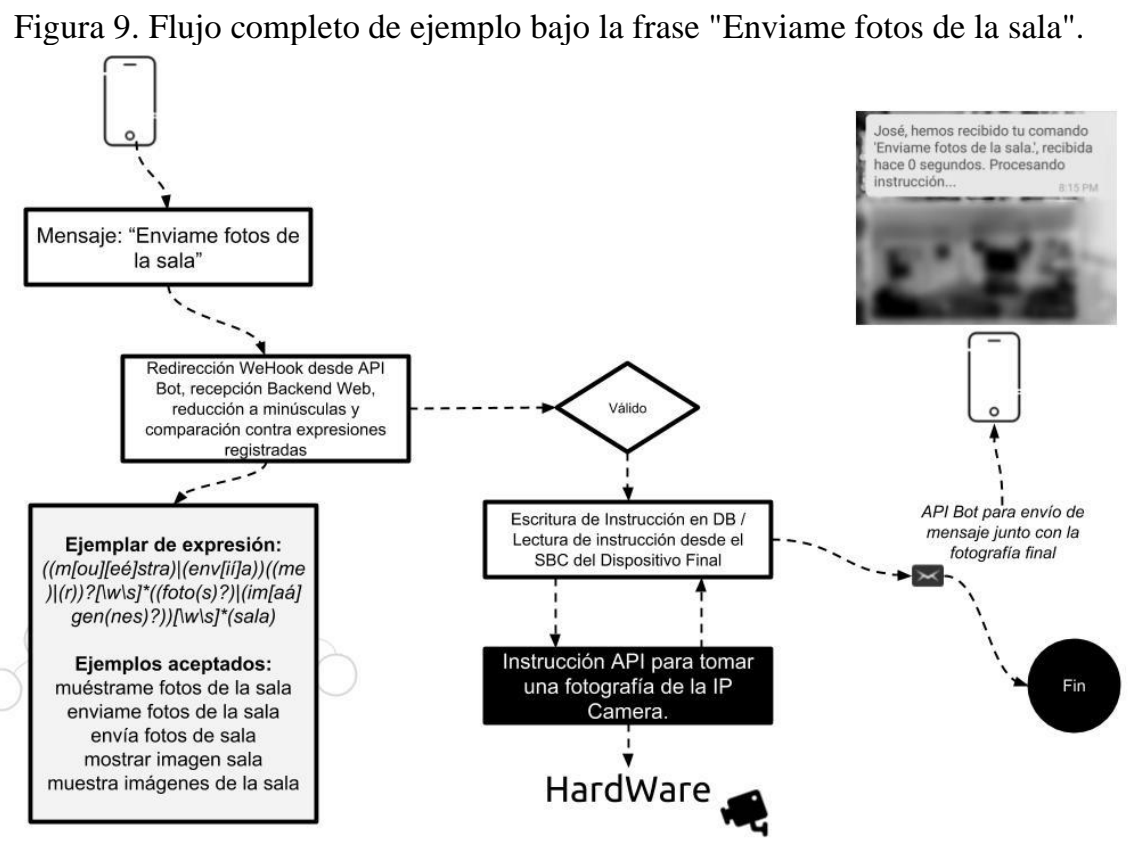

\section{G. Integración con IFTTT como Software para IoT}

Existe un software en línea llamado IFTTT ("IF This Then That" o "Si esto, entonces aquello" para su significado en español) [10] asociado con numerosos servicios (Gmail, WikiPedia, DropBox, Pocket, Youtube, Trello, Giphy, etc) y protocolos (SMS's, GPS, SMTP's, Rss, HTTP, etc) en internet para ofrecer conectividad hacia IoT que aprovecha un ecosistema integrado entre compañías, aplicaciones y dispositivos para interactuar con sensores, ubicación, servicios y consultas en internet para la generación de acciones.

Para unificar los conceptos (Gadgets, Home Appliances, Related IoT y IFTTT Services), la Fig. 9 plantea el agrupamiento de todos estos mensajes, al hacer uso de los Grupos de Telegram, para integrar el Bot de IFTTT y nuestro propio Bot en una única interfaz de texto para recibir textos o multimedia de servicios de software en línea alimentados por IFTTT como también de nuestros propios desarrollos en casa conectados por nuestro BackEnd Web. 
Figura 10. Diseño de unificación de mensajes entre Bots de Telegram, IFTTT y un BackEnd Web de servicios propios con mensajes de texto para entregar instrucciones en lenguaje Natural.

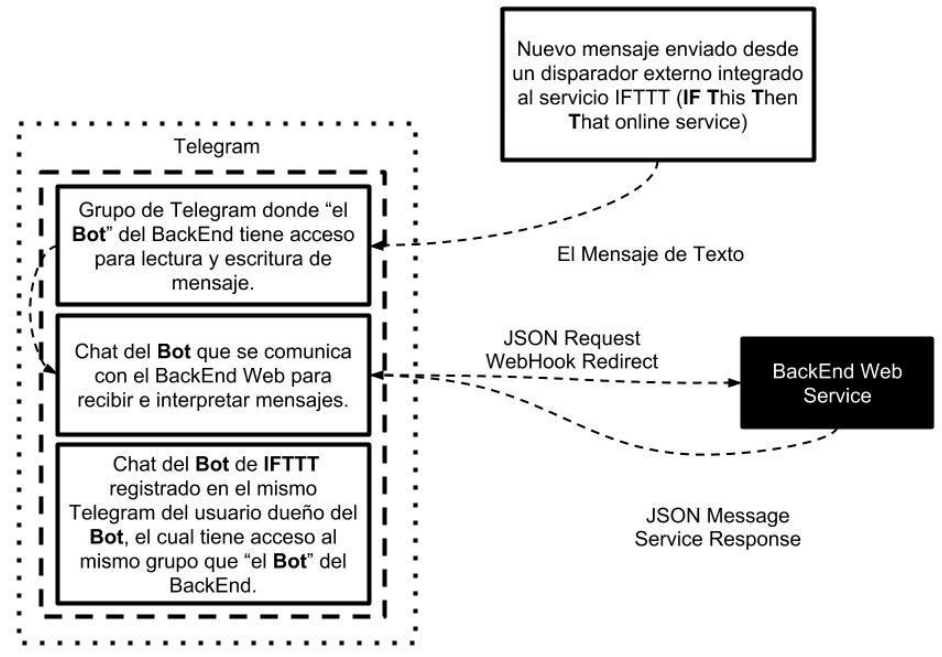

Para utilizar IFTTT el usuario debe cursar por los pasos de registro para el uso gratuito de la plataforma, lo que le permitirá configurar "applets". Un applet es una conexión entre dos aplicaciones o dispositivos, logrando acciones que una app por si sola no podría hacer. Los applets se componen de triggers o disparadores, y de actions o acciones. El disparador le dice al applet cuando iniciar y las acciones son el resultado final de la ejecución del applet. Es este mismo concepto usado por IFTTT el que nos permitirá en nuestras propias implementaciones, realizar la ejecución de una acción al recibir las instrucciones en BackEnd Web pero que en lugar de usarlo para conectarse con apps de la web, se usará para comunicar una acción al API o la interfase de nuestros periféricos personales o en casa conectados a internet.

\section{H. Consideraciones de Seguridad Informática}

El potencial riesgo de pérdida de información al comprometer nuestros propios dispositivos de casa a través de Internet, siempre estará presente mientras existan múltiples partes y componentes involucrados. Todo el software o hardware que usamos proviene, se ejecuta o reside bajo lineamientos o características de distintos fabricantes, se encuentran expuestos ante una gran cantidad de usuarios y esto expande las posibilidades de encontrar problemas de seguridad, sea sobre lenguajes de programación, sistemas operativos, servicios, micro-procesadores, micro-controladores $\mathrm{u}$ otros componentes. Únicamente con encontrar una vulnerabilidad dentro de alguna de estas partes, el sistema como un conjunto compartirá el mismo potencial de riesgo pero aun así es posible seguir lineamientos particulares enfocados a la mitigación de problemas de seguridad que reducen las brechas dentro de los componentes que están bajo nuestro control. 
Disponer de un servicio centralizado privado (nuestro agente intermediario en la nube que alimenta de respuestas al Bot y que lee la entrada de datos de texto del usuario final) no asegura implícitamente protección, sin embargo, contribuye a centralizar dentro del mismo servicio múltiples capas de seguridad para compartir la misma protección entre diferentes partes del sistema. Esta ventaja permitirá al implementador enfocar la mayoría de esfuerzo en esta única plataforma integrada sin perder de vista el resto de componentes.

Bajo el criterio experimental antes expuesto, para habilitar un servicio Bot para comunicarse con dispositivos IoT se recomienda tomar las siguientes consideraciones esenciales: 1) Hacer uso de SSL (Secure Socket Layer por sus siglas en inglés) para los servicios conectados, mediante implementación de un agente HTTPS para el WebHook entre el Bot de la plataforma de chat y el software de interpretación en la nube, además de implementar el mismo protocolo dentro de cada SBC intermediaria. 2) Evitar los comandos "toggle switching" y uso de cola (primero en llegar primero en salir) donde al hacer el primer llamado el dispositivo se enciende mientras que el segundo llamado el dispositivo se apaga. Esta modalidad puede ser arriesgada en redes con posible delay o latencia al intentar comunicarse con el servicio final. Transportar instrucciones bajo el criterio de cola podría provocar duplicidad de comandos, que al mismo tiempo pueden provocar activaciones y desactivaciones masivas y consecutivas a "velocidad del circuito" lo que puede significar daño permanente en los componentes al encenderlos y apagarlos constantemente en extrema velocidad. En su lugar, la lógica del agente intermediario debe controlar si registra o no la instrucción recibida al compararla con el estado actual del dispositivo. 3) Contar con infraestructura de alta disponibilidad contratando servidores en la nube en lugar de mantener el servidor intermediario y los periféricos bajo la misma red, cuando el propósito final es acceder a estos a través de internet. De esta forma si nuestro agente en la web controla más de un hogar o interconecta dispositivos fuera del alcance de la red del hogar, estos igual podrá seguir en funcionamiento ya que dependen de redes independientes. Además de esta forma reducimos los costos de mantenimiento eléctrico, de actualizaciones de seguridad de sistema operativo y de otros costos y tareas operativas que absorben las granjas de servidores en Internet. 4) Hacer uso de red cableada sobre red inalámbrica cuando sea posible para los dispositivos en casa bajo una configuración NAT separada e inaccesible a la red Wi-Fi. De esta forma nuestro servicio podrá accederse de una manera aislada de la red principal. 5) Evitar el uso de componentes de software y hardware deprecados, desactualizados u obsoletos para reducir el riesgo de brechas de seguridad por falta de parches ante bugs universales. 6) Proteger con autenticación de Usuario y Clave cada servicio que se comunique con el servicio externo cuando sea posible (como por ejemplo los accesos para lectura y escritura de base de datos) y mantener un alto estándar de definición y protección de estos datos en todo momento. 


\section{CONCLUSIONES}

Es posible usar el modelo de Bot para implementar una red de comunicación entre dispositivos de IoT hechos en casa o de un fabricante tercero. Las limitantes de cada servicio estarán definidas por el API de uso de cada dispositivo independiente sin embargo la implementación de este modelo es posible bajo múltiples escenarios. La matriz de acciones que el investigador pueda implementar dentro del agente intermediario para poder enviar instrucciones al periférico final se limita a las acciones que se desean realizar a través del comando del texto que recibirá el Bot, junto con las posibilidades que entregue mediante software y hardware cada fabricante sobre sus propios dispositivos IoT, y de la complejidad o simplicidad a la que se enfrente el implementador para interconectar dispositivos mecánicos, eléctricos o electrónicos que no están preparados para IoT.

La complejidad o simplicidad de los sistemas no alejan a los componentes de hardware o software involucrados de ser vulnerables ante brechas de seguridad. El acercamiento que muestra este estudio sobre la conectividad de dispositivos para establecer una comunicación unificada de instrucciones a través de texto implica que existen numerosos puertos, servicios, computadores y accesos involucrados en los enlaces, en el envío y recepción de mensajes, en la autenticación entre sistemas y en el manejo de nuestros aparatos domésticos, lo que podría generar por tanto brechas importantes de seguridad si no se manejan bajo estándares o mecanismos conocidos como buenas prácticas. Para contrarrestar estas posibles brechas que están bajo nuestro control, el implementador deberá mantener bajo anonimato datos de conectividad como direcciones IP, URL's de servicio, claves API, usuarios y contraseñas, además de hacer uso de protocolo SSL para todas las comunicaciones en Internet. Además, controlar bajo configuración del servicio API del Bot y bajo programación del agente intermedio el acceso a los servicios sobre usuarios específicos de tal forma que otros usuarios no puedan acceder a dicho Bot privado, ni a la plataforma que se ha construido detrás del él.

Al unificar múltiples servicios dentro de una única interfaz familiar de texto, reducimos la curva de aprendizaje para que otros usuarios menos experimentados puedan acceder a un rango mayor y más personalizado de dispositivos interconectados a Internet. En lugar de limitarse a utilizar únicamente dispositivos comerciales de IoT, y aunque dependemos de cada fabricante para que provea de las herramientas necesarias para poder acceder a sus productos de otras maneras distintas, otros usuarios en nuestros propios hogares pordían involucrarse con un uso más sencillo y personalizado de realizar actividades convencionales como apagar las luces, encender la ventilación o de revisar una cámara de seguridad. No todos los usuarios comparten el mismo nivel de experiencia en el uso de tecnologías e implementar una solución centralizada como la definida en este estudio, podría representar una manera más familiar usar dispositivos inteligentes. ¿Cuántas personas conocemos en nuestro círculo cercano sabe 
usar un chat? Aunque la respuesta no sea "la totalidad de personas", los hábitos y necesidades de generaciones actuales pueden sentirse más en sintonía con esta forma de comunicación entre dispositivos.

Basta con usar programación tradicional por procedimientos para la implementación propuesta por este estudio. Aunque podemos hacer uso de tecnologías de inteligencia artificial para incrementar el número de escenarios a reconocer, usando programación tradicional de comparación de casos bajo un esquema simple como el de Expresiones Regulares o con el uso de Árboles de Decisión, es suficiente para usar este modelo de comunicación con dispositivos de Internet de las Cosas. El modelo general de condición/evento/trigger que inicia nuestro proceso inteligente, y la ejecución/acción/notificación que finaliza la tarea, corresponde con la funcionalidad general de entrada, proceso y salida básicos que componen todo diseño de "caja negra" de los sistemas informáticos. Este simple modelo define que la entrada corresponde con una llamada provocada por un evento pre-programado cualquiera; el proceso recibe e interpreta el contenido de la instrucción escrita, toma las decisiones y transforma la información recibida según sus condiciones establecidas y así la salida cumple la tarea solicitada. Sumando lo anterior al modelo de texto ordenado según patrones de oraciones representa una forma de traducir complejos algoritmos de programación en simples cadenas de expresiones que probablemente sea más fácil de implementar por lectores o investigadores que estén familiarizados con tecnologías y metodologías similares a las expuestas en este documento bajo el fenómeno estudiado.

\section{AGRADECIMIENTOS}

Haciendo uso de este medio comparto mi agradecimiento más sincero hacia nuestro Divino Creador siempre presente en nuestras vidas, a mi Esposa (E. R.) como fuente de apoyo e inspiración, a mi familia cercana (A.M., R.I, D. A., J. R., A.I, S.S, V. S. I. S., M. V. I. S., M. I., M. S., S. I., S. P., S. M. C.) y amistades indispensables (M. L., W. M. y F. L.).

A los emprendedores de servicios gratuitos y abiertos en internet que proveen de plataformas, recursos y software para generar ideas en beneficio científico y social, sean grandes o pequeñas corporaciones.

A todo aquel que avanza inclusive cuando no es posible tomar impulso.

A todos aquellos "que son esa clase de hombres que nacen con la boca y la voz en la mano", para comunicar sus ideas en pro de la humanidad (A.V.). 


\section{REFERENCIAS BIBLIOGRÁFICAS}

A.M. Turing, "Mind - A Quarterly Review Of Psychology And Philosophy" o "Mente - Una visión parcial de la Psicología y Filosofía”, VOL. LIX. NO. 236, p. 1, October 1950

P. Barnaghi, W. Wang, C. Henson, K. Taylor,"Semantics for the Internet of Things: early progress and back to the future", "Why Semantics Are Important?", pp. 3 - 4, 2012

P.-L. Tu ; J.-Y. Chung, “A new decision-tree classification algorithm for machine learning” o "Un nuevo algoritmo de clasificación con árboles de decisión para aprendizaje máquina”, November 1992

S. Palaniappan, N. Hariharan, N. T. Kesh, Vidhyalakshimi S., A. Deborah S., "Home Automation Systems - A Study", Volume 116 - No. 11, April 2015

E. White (O'Reilly), "Making Embedded Systems”, p. 1, 2011

V. Sathya Narayanan, S.Gayathri, "Design of Wireless Home automation and security system using PIC Microcontroller”, Volume III, Special Issue, August 2013

A. Gómez, D. Cuiñas, P. Catalá, L. Xin, W. Li, S. Conway, D. Lack, "Use of Single Board Computers as Smart Sensors in the Manufacturing Industry" o "Uso de computadores de una sola placa como sensores inteligentes en la industria de la manufactura", Procedia Engineering, pp. 153 - 155, 2015

S. Reddy, O. Tackstr, S. Petrov, M. Steedman, M. Lapata,“Universal Semantic Parsing” o "Parseo Universal de Semántica", p. 2, 2017.

Telegram, “Core Bots API”, https://core.telegram.org/bots/api

“IFTTT - IF This Then That”, https://platform.ifttt.com/

R. H. Weber, Romana Weber, "Internet of Things Legal Persepectives" o "Internet de las Cosas Perspectiva Legal", 2010.

A. Bejarano, A. Fernández, M. Jimeno, A. Salazar, P. Wightman, "Towards the Evolution of Smart Home Environments: A Survey" o "Hacia la Evolución de los Ambientes de Casas Inteligentes: Una Encuesta", Original Article auSMT Vol. 6 No.3, pp. 106 - 110, 2016.

D. Goldwasser, D. Roth, "Learning From Natural Instructions", University of Illinois at UrbanaChampaign, pp. 1 - 2.

ADNAN. A. Arain, A. Manzoor, K. Brohi, K. Haseeb, I. A. Halepoto, I. A. Korejo,"Artificial Intelligence Mark-up Language Based Written and Spoken Academic Chatbots using Natural Language Processing", Vol.50 (001), pp.153-155, 2017-2018. 